\title{
De l'efficacité des dépenses publiques d'éducation en Amérique latine
}

\author{
par Pablo Zoido
}

\section{(D'après Perspectives économiques de l'Amérique latine 2009)}

www.oecd.org/dev/publications/leo2009

- Les dépenses publiques d'éducation sont en hausse en Amérique latine, mais les avancées récentes ne peuvent masquer des problèmes persistants.

- Les systèmes éducatifs latino-américains doivent être plus inclusifs et l'enseignement de meilleure qualité.

L'enseignement est l'un des principaux moteurs de la croissance économique. Il apporte non seulement un savoir théorique, mais contribue aussi à la réalisation d'objectifs économiques, tels que la croissance et la productivité, et sociaux, comme la santé et la cohésion sociale. Dans une économie mondiale fortement concurrentielle, les dépenses publiques d'éducation sont plus importantes que jamais.

D'après Perspectives économiques de l'Amérique latine 2009 de I'OCDE, les dépenses publiques d'éducation sont importantes et en augmentation dans la région. En pourcentage du PIB ou des dépenses publiques totales, elles y sont aussi élevées que dans les pays de I'OCDE. Cependant, en moyenne, I'Amérique latine dépense encore cinq fois moins d'argent par élève que n'importe quel pays de I'OCDE. L'explication est en partie démographique, car la population en âge d'être scolarisée y est beaucoup plus nombreuse que dans les pays de I'OCDE. Mais les disparités entre les pays latino-américains et la zone OCDE restent marquées, même en termes relatifs : ainsi, les dépenses par élève dans l'enseignement secondaire représentent 13 pour cent du PIB dans la région, soit à peine la moitié du chiffre de I'OCDE.

Malgré des pressions démographiques significatives, les gouvernements des pays de la région ont néanmoins réussi à ce que la plupart des habitants reçoivent au moins une éducation de base : l'inscription dans le primaire est quasiment universelle dans la région, et la proportion des plus de 15 ans qui n'ont jamais été scolarisés est aujourd'hui tombée à 6 pour cent seulement. Si les taux d'inscription augmentent globalement, de profonds écarts demeurent audelà du primaire, en particulier pour les ménages pauvres.
Ainsi, au Nicaragua, plus de 70 pour cent des enfants de ménages riches fréquentent l'école secondaire, contre moins de 15 pour cent des enfants pauvres.

L'augmentation des dépenses n'est pas forcément synonyme d'amélioration de la qualité. Les pays d'Amérique latine ne semblent pas tirer le meilleur parti des dépenses qu'ils engagent. Par exemple, les systèmes éducatifs de Lituanie et de Macao-Chine consacrent autant de moyens par élève que ceux d'Amérique latine, mais les élèves de ces deux pays obtiennent de meilleurs résultats aux tests standardisés, quelle que soit leur classe socio-économique. D’après les conclusions de l'enquête PISA 2006 (Programme international pour le suivi des acquis des élèves) de I'OCDE, les LatinoAméricains de 15 ans affichent en moyenne l'équivalent de trois années de scolarité de retard sur leurs homologues de I'OCDE. Dans les autres pays émergents, les élèves obtiennent eux aussi des scores inférieurs à ceux de l'OCDE, mais l'écart ne représente que la moitié de celui qui ressort de l'enquête pour les six pays d'Amérique latine. Plus inquiétant, les taux d'échec scolaire sont très élevés chez les élèves latino-américains. Selon l'évaluation PISA 2006, la majorité des étudiants de la région ne possèdent pas les bases pour la compréhension de l'écrit.

Ainsi, malgré des progrès impressionnants, I'amélioration de la qualité de l'enseignement et du taux de participation et de persévérance scolaire des élèves reste l'un des principaux obstacles au développement de l'Amérique latine. Les performances sont globalement en progression, mais restent faibles par rapport aux autres pays. Selon l'enquête PISA, les pays d'Amérique latine mettent actuellement insuffisamment I'accent sur certaines des politiques d'éducation qui semblent les plus importantes pour l'apprentissage des élèves. 
Figure 1. Dépenses publiques d'éducation et résultats PISA

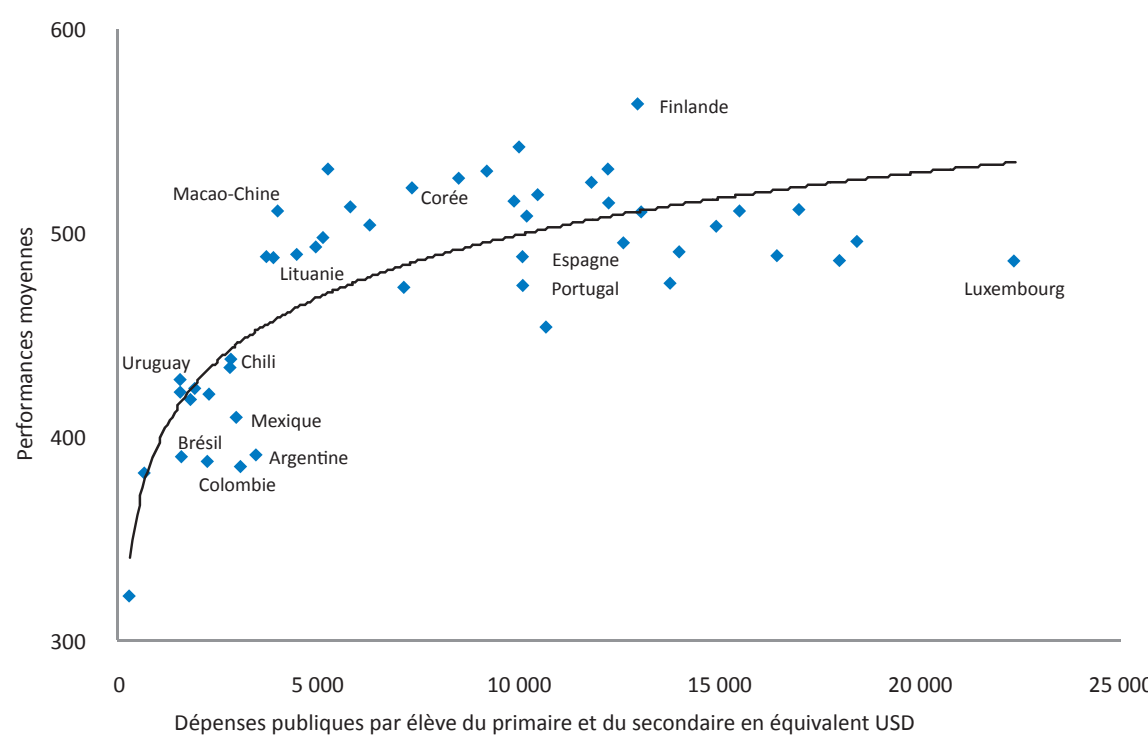

Note : Les performances sont mesurées par la moyenne du pays en matière de culture scientifique de l'étude PISA 2006. Les dépenses publiques par élève dans l'enseignement primaire et secondaire correspondent à la moyenne des données disponibles depuis 2000.

Source : Perspectives économiques de l'Amérique latine 2009, OCDE 2008.
Si l'on renforce la transparence et encourage les élèves à consacrer davantage de temps à leurs leçons et à d'autres activités propices à l'apprentissage, on s'aperçoit que les performances semblent s'améliorer. Autre constat important qui ressort des évaluations internationales telles que le PISA : il n'y a pas d'arbitrage automatique entre qualité des performances et équité. Les pays qui obtiennent les meilleurs scores sont ceux qui encouragent l'apprentissage dans tous les segments socioéconomiques de la population.

L'éducation constitue un excellent exemple des problèmes que rencontre l'Amérique latine alors qu'elle cherche plus généralement à améliorer sa politique budgétaire. Il faudrait non seulement accroître les moyens financiers, mais aussi, et surtout, savoir comment ils vont être dépensés. Les réformes actuellement menées au Chili et au Mexique montrent bien que chaque cas nécessite une solution adaptée au système éducatif et au contexte politique du pays en question.
Les lecteurs sont invités à citer ou reproduire les informations des Repères du Centre de développement de I'OCDE dans leurs propres publications. En échange, le Centre demande les remerciements de rigueur ainsi qu'un exemplaire de la publication. Le texte intégral des Repères et d'autres informations sur le Centre de développement et ses travaux sont disponibles sur : www.oecd.org/dev
Centre de développement de I'OCDE 2, rue André-Pascal, 75775 Paris Cedex 16, France Tél : $33(0) 145.24 .82 .00$ Fax : $33(0) 144.30 .61 .49$ mél : cendev.contact@oecd.org 